\title{
BM]
}

\section{Impact of self monitoring of blood glucose in the management of patients with non-insulin treated diabetes: open parallel group randomised trial}

\author{
Andrew Farmer, lecturer, ${ }^{1}$ Alisha Wade, resident, ${ }^{2}$ Elizabeth Goyder, reader, ${ }^{3}$ Patricia Yudkin, reader, ${ }^{1}$ David \\ French, reader, ${ }^{4}$ Anthea Craven, trial manager, ${ }^{1}$ Rury Holman, professor, ${ }^{5}$ Ann-Louise Kinmonth, professor, ${ }^{6}$ \\ Andrew Neil, professor, ${ }^{7}$ on behalf of the Diabetes Glycaemic Education and Monitoring Trial Group
}

'Department of Primary Health

Care, University of Oxford, Oxford OX2 7LF

${ }^{2}$ Johns Hopkins University School of Medicine, Baltimore

${ }^{3}$ School of Health and Related Research, University of Sheffield

${ }^{4}$ Applied Research Centre in Health and Lifestyle Interventions, University of Coventry

${ }^{5}$ Diabetes Trials Unit, University of Oxford

${ }^{6}$ Institute of Public Health, University of Cambridge

${ }^{7}$ Division of Public Health and Primary Care, University of Oxford

Correspondence to: A Farmer andrew.farmer@dphpc.ox.ac.uk

doi:10.1136/bmi.39247.447431.BE

\section{ABSTRACT}

Objective To determine whether self monitoring, alone or with instruction in incorporating the results into self care, is more effective than usual care in improving glycaemic control in non-insulin treated patients with type 2 diabetes.

Design Three arm, open, parallel group randomised trial. Setting 48 general practices in Oxfordshire and South Yorkshire.

Participants 453 patients with non-insulin treated type 2 diabetes (mean age 65.7 years) for a median duration of three years and a mean haemoglobin $\mathrm{A}_{1 \mathrm{c}}$ level of $7.5 \%$. Interventions Standardised usual care with measurements of $\mathrm{HbA}_{1 \mathrm{c}}$ every three months as the control group $(n=152)$, blood glucose self monitoring with advice for patients to contact their doctor for interpretation of results, in addition to usual care $(n=150)$, and blood glucose self monitoring with additional training of patients in interpretation and application of the results to enhance motivation and maintain adherence to a healthy lifestyle ( $n=151)$.

Main outcome measure $\mathrm{HbA}_{1 \mathrm{c}}$ level measured at 12 months.

Results At 12 months the differences in $\mathrm{HbA}_{1 \mathrm{c}}$ level between the three groups (adjusted for baseline $\mathrm{HbA}_{1 \mathrm{c}}$ level) were not statistically significant $(P=0.12)$. The difference in unadjusted mean change in $\mathrm{HbA}_{1 \mathrm{c}}$ level from baseline to 12 months between the control and less intensive self monitoring groups was $-0.14 \%$ (95\% confidence interval $-0.35 \%$ to $0.07 \%$ ) and between the control and more intensive self monitoring groups was $-0.17 \%(-0.37 \%$ to $0.03 \%)$.

Conclusions Evidence is not convincing of an effect of self monitoring blood glucose, with or without instruction in incorporating findings into self care, in improving glycaemic control compared with usual care in reasonably well controlled non-insulin treated patients with type 2 diabetes.

Trial registration Current Controlled Trials ISRCTN47464659.

\section{INTRODUCTION}

As the number of people diagnosed as having type 2 diabetes continues to rise ${ }^{1}$ so does the challenge of monitoring and managing the condition. Self monitoring of blood glucose for people with non-insulin treated diabetes may lead to improved glycaemic control and is commonly recommended. Existing evidence of effectiveness is, however, inconclusive..$^{2-4}$ Despite limitations in trial evidence, proponents of self monitoring of blood glucose cite the benefits reported in some observational studies of patients with diabetes, ${ }^{56}$ but these analyses may not have fully adjusted for the potential confounding effect of an association between self monitoring of blood glucose and take-up of other health improving behaviours. Meanwhile the use of self monitoring in this group of patients and the cost to health systems of the consumable test strips have become a major and increasing proportion of healthcare budgets. $^{78}$

We tested whether self monitoring of blood glucose, with or without instruction in incorporating findings into self care, compared with standardised usual care can improve glycaemic control in patients with noninsulin treated diabetes.

\section{METHODS}

The diabetes glycaemic education and monitoring (DiGEM) study was a four year open, randomised, three arm, parallel group trial with sequential recruitment of patients from general practices in Oxfordshire and South Yorkshire. The trial was managed from the coordinating centre at the Department of Primary Health Care, University of Oxford. ${ }^{9}$

Our primary aim was to determine whether haemoglobin $\mathrm{A}_{1 \mathrm{c}}\left(\mathrm{HbA}_{1 \mathrm{c}}\right)$ levels at 12 months were significantly different between patients with non-insulin treated type 2 diabetes receiving one of three allocated interventions: standardised usual care with measurements of $\mathrm{HbA}_{1 \mathrm{c}}$ levels by health professionals every three months (control group); use of a blood glucose meter, with advice for participants to contact their doctor for interpretation of results (less intensive self monitoring); and use of a blood glucose meter with training in self interpretation and application of the results to diet, physical activity, and drug adherence (more intensive self monitoring). 


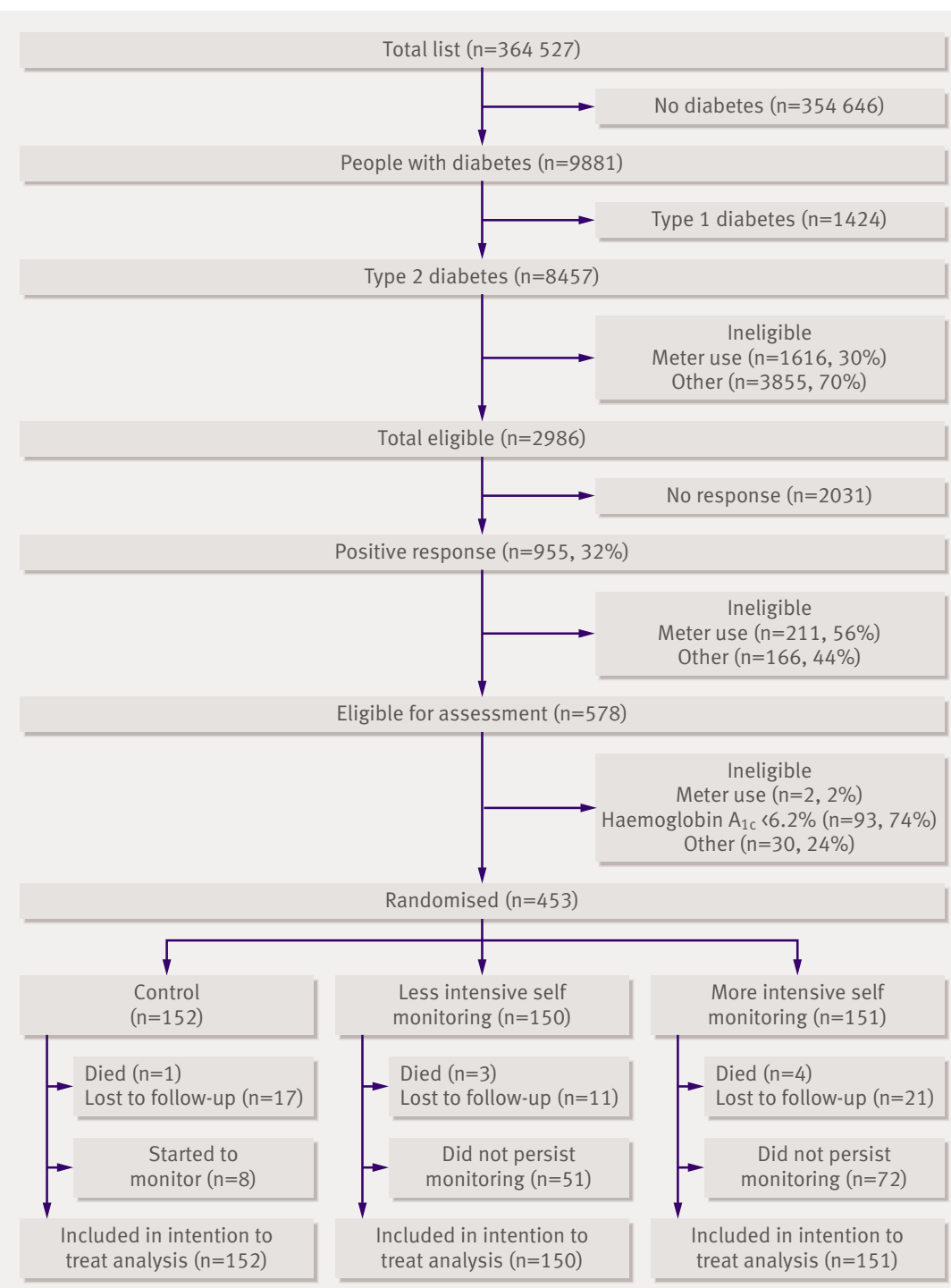

Fig 1 | Trial profile standardisation programme and comparable to the diabetes control and complications trial standard, with an interassay coefficient of variation across the range of the assay of less than $2 \%$. Cholesterol was assayed in local laboratories and the results aligned with results of a sample of paired specimens analysed with an automated chemistry analyser (Olympus AU400; Olympus, Tokyo), with interassay coefficients of variation across the range of less than $2 \%$. Blood pressure was measured twice in the right arm, with the participant seated, using a UA-779 electronic blood pressure monitor (A\&D instruments, Abingdon), and the mean of these values was analysed.

We transcribed the frequency of blood glucose testing from patient held diaries. Episodes of hypoglycaemia were categorised as grade 2 (mild symptoms requiring minor intervention), grade 3 (moderate symptoms requiring immediate third party intervention), or grade 4 (unconscious). Increases in hypoglycaemic drugs were defined as an increase in the dose or frequency prescribed, progression from use of a single oral agent to combination oral therapy, or addition of insulin to the treatment regimen.

To characterise the groups and identify subgroups for predefined analysis at 12 months we collected additional personal and clinical data on duration of diabetes, drug treatment, diabetes related complications, and EuroQol (EQ-5D) score. ${ }^{10}$

\section{Randomisation}

We used computerised randomisation (Minim, www. sghms.ac.uk/depts/phs/guide/randser.htm) incorporating a partial minimisation procedure to adjust the randomisation probabilities between groups to balance three important covariates collected at baseline: duration of diabetes, $\mathrm{HbA}_{1 \mathrm{c}}$ level, and current treatment (diet, oral monotherapy, or oral combination therapy). The minimisation procedure to assign patients to their allocated intervention was conducted independently of the research nurses who managed recruitment and carried out the assessment visit. The allocation was also concealed from laboratory staff.

Patients were eligible for randomisation if they had type 2 diabetes, were aged 25 years or more at diagnosis, were managed with diet or oral hypoglycaemic agents alone, had an $\mathrm{HbA}_{1 \mathrm{c}}$ level $\geq 6.2 \%$ at the assessment visit, and were independent in activities of daily living. Exclusion criteria were the use of a blood glucose monitor twice a week or more often over the previous three months, serious disease or limited life expectancy that would make intensive glycaemic control inappropriate, or inability to follow trial procedures.

\section{Outcome measures}

The primary outcome was the $\mathrm{HbA}_{1 \mathrm{c}}$ level at 12 months. Secondary outcomes were blood pressure, weight, total cholesterol level, ratio of total cholesterol to high density lipoprotein cholesterol, and body mass index. $\mathrm{HbA}_{1 \mathrm{c}}$ was measured using a Variant II Hemoglobin Testing System (Bio-Rad Laboratories, Hercules, CA) certified by the US glycohaemoglobin

\section{Procedures}

We identified patients suitable for trial inclusion from lists held on computer by their general practitioners. Those eligible were sent an invitation to participate signed by their general practitioner accompanied by an information sheet and a reply paid envelope. One further letter was sent if no response was received within one month.

Eligibility for the trial and willingness to be randomised to self testing of blood glucose was confirmed by a preassessment phone call and at the visit for assessment. At the assessment visit, after obtaining informed consent, beliefs about diabetes were elicited using a standard approach to help patients understand how diabetes might present a threat to their health. ${ }^{11}$ The roles of diet, physical activity, and drugs were discussed within the framework of the commonsense model of illness representation, ${ }^{11}$ in which we set out to optimise the use of feedback on glucose levels to 
facilitate behaviour change through influencing beliefs. The behaviour change techniques were selected on the basis of evidence for effectiveness and included goal setting and review of physical activity and eating patterns to help patients with lifestyle change. ${ }^{12} 13$ The goal setting and review approach was continued in subsequent visits. Baseline blood tests and clinical measurements were taken and questionnaires completed at the assessment visit.

\section{Interventions}

After the assessment visit and confirmation of eligibility, patients were allocated to receive one of the three interventions. The rationale behind these interventions is described in more detail elsewhere. ${ }^{9}$ The

Table 1 | Personal and baseline characteristics of patients with non-insulin treated type 2 diabetes before intervention, by randomisation group. Values are numbers (percentages) unless stated otherwise

\begin{tabular}{|c|c|c|c|}
\hline Variables & $\begin{array}{l}\text { Control group* } \\
\quad(n=152)\end{array}$ & $\begin{array}{l}\text { Meter group; less } \\
\text { intensive self } \\
\text { monitoring }(n=150)\end{array}$ & $\begin{array}{l}\text { Meter group; more } \\
\text { intensive self } \\
\text { monitoring }(n=151)\end{array}$ \\
\hline \multicolumn{4}{|l|}{ Personal characteristics } \\
\hline Mean (SD) age (years) & $66.3(10.2)$ & $65.2(10.6)$ & $65.5(9.9)$ \\
\hline Men & $85(55.9)$ & $88(58.7)$ & $87(57.6)$ \\
\hline \multicolumn{4}{|l|}{ Occupational group: } \\
\hline $\begin{array}{l}\text { Professional, managerial, and } \\
\text { clerical }\end{array}$ & $80(52.6)$ & $81(54.0)$ & $84(55.6)$ \\
\hline Skilled manual or manual & $69(45.4)$ & $68(45.3)$ & $66(43.7)$ \\
\hline No occupation stated & $3(2.0)$ & $1(0.7)$ & $1(0.7)$ \\
\hline
\end{tabular}

Age (years) at leaving full time

education:

\begin{tabular}{|c|c|c|c|}
\hline$\ll 17$ & $109(71.7)$ & $114(76.0)$ & $121(80.1)$ \\
\hline 17 or 18 & $20(13.2)$ & $14(9.3)$ & $13(8.6)$ \\
\hline$>18$ & $23(15.1)$ & $22(14.7)$ & $17(11.3)$ \\
\hline \multicolumn{4}{|l|}{ Cigarette consumption: } \\
\hline Never smoked & $58(38.2)$ & $54(36.2)$ & $54(35.8)$ \\
\hline Former smoker & $80(52.6)$ & $74(49.7)$ & $77(51.0)$ \\
\hline Current smoker & $14(9.2)$ & $21(14.1)$ & $20(13.2)$ \\
\hline \multicolumn{4}{|l|}{ Duration of diabetes and treatment } \\
\hline $\begin{array}{l}\text { Median (interquartile range) } \\
\text { duration (years) of diabetes }\end{array}$ & $3(2-6)$ & $3(2-7)$ & $3(2-6)$ \\
\hline \multicolumn{4}{|l|}{ Treatment: } \\
\hline Diet only & $44(28.9)$ & $39(26.0)$ & $41(27.2)$ \\
\hline Monotherapy & $57(37.5)$ & $58(38.7)$ & $58(38.4)$ \\
\hline Combined oral therapy & $51(33.6)$ & $53(35.3)$ & $52(34.4)$ \\
\hline $\begin{array}{l}\text { Presence of diabetes related } \\
\text { complications }\end{array}$ & $32(21.1)$ & $32(21.3)$ & $39(25.8)$ \\
\hline \multicolumn{4}{|l|}{ Use of blood glucose meter: } \\
\hline Not using & $104(68.4)$ & $110(73.3)$ & $102(67.5)$ \\
\hline Using once weekly or less & $48(31.6)$ & $40(26.7)$ & $49(32.5)$ \\
\hline \multicolumn{4}{|l|}{ Physical and laboratory findings } \\
\hline Mean (SD) haemoglobin $\mathrm{A}_{1 \mathrm{c}}(\%)$ & $7.49(1.09)$ & $7.41(1.02)$ & $7.53(1.12)$ \\
\hline $\begin{array}{l}\text { Mean (SD) total cholesterol level } \\
(\mathrm{mmol} / \mathrm{l})\end{array}$ & $4.7(1.1)$ & $4.6(1.1)$ & $4.7(1.1)$ \\
\hline \multicolumn{4}{|l|}{$\begin{array}{l}\text { Mean (SD) blood pressure (mm } \\
\mathrm{Hg}):\end{array}$} \\
\hline Systolic & $140(18)$ & $141(17)$ & $137(18)$ \\
\hline Diastolic & $80(10)$ & $80(10)$ & $78(10)$ \\
\hline Mean (SD) body mass index & $30.9(6.1)$ & $31.9(6.2)$ & $31.0(5.3)$ \\
\hline
\end{tabular}

*No use of blood glucose meter. intervention was initiated at the first visit after randomisation and continued at the scheduled visits at one, three, six, and nine months. Each of the three interventions included a series of standardised components.

Patients allocated to the control intervention received standardised usual care, including the use of goal setting and review. They were asked not to use a blood glucose meter unless their doctor considered it essential for their clinical management. A diary was used to record self care goals and strategies for achieving them.

Patients allocated to the less intensive self monitoring intervention continued to use the goal setting and review techniques introduced at the assessment visit. In addition they were given a blood glucose meter. They were asked to record three values daily on two days during the week (one after fasting and the other two before meals or two hours after meals) and to aim for glucose levels of 4-6 mmol/l after fasting and before meals and levels of 6-8 mmol/1 two hours after meals. They were advised by the nurse to consider contacting their doctor if readings were consistently high $(>15 \mathrm{mmol} / \mathrm{l})$ or low $(<4 \mathrm{mmol} / \mathrm{l})$. They were not given information about how to interpret their blood glucose readings. Separate diaries were used to record identified goals and activity and to record blood glucose results.

Patients allocated to the more intensive intervention continued to use goal setting and review and were also given a blood glucose meter. They were also given training and support in timing, interpreting, and using the results of their blood glucose test to enhance motivation and to maintain adherence to diet, physical activity, and drug regimens. They were encouraged to experiment with monitoring to explore the effect of specific activities, such as exercise, on their blood glucose level and to reflect on abnormal values in an attempt to identify what might have contributed to them. A single diary was used to record goals, activities, and blood glucose results.

Follow-up visits differed in content according to the allocated intervention in line with usual practice. Patients allocated to the control intervention had a blood test to measure $\mathrm{HbA}_{1 \mathrm{c}}$ level two weeks before their scheduled visit, the result of which was fed back to them as an indication of the impact of their self care activities on their glycaemic control. Blood glucose values were reviewed at the scheduled visit for those allocated to self monitoring, and patients were told to seek advice from their doctor if fasting values were persistently greater than $6 \mathrm{mmol} / \mathrm{l}$. Patients in each arm of the trial received feedback on glycaemic control, which was used to explore success of goals and to set new ones. The patient's doctor was notified of all $\mathrm{HbA}_{1 \mathrm{c}}$ results and asked to consider changes in drugs in line with the National Institute for Clinical Excellence diabetes guidelines for all patients. ${ }^{14}$ The doctor was also notified if blood glucose readings were consistently greater than $15 \mathrm{mmol} / \mathrm{l}$.

The meters were calibrated to provide plasma equivalent results (Optium, Abbott Diabetes Care, Maidenhead, UK). Calibration of the meters was 


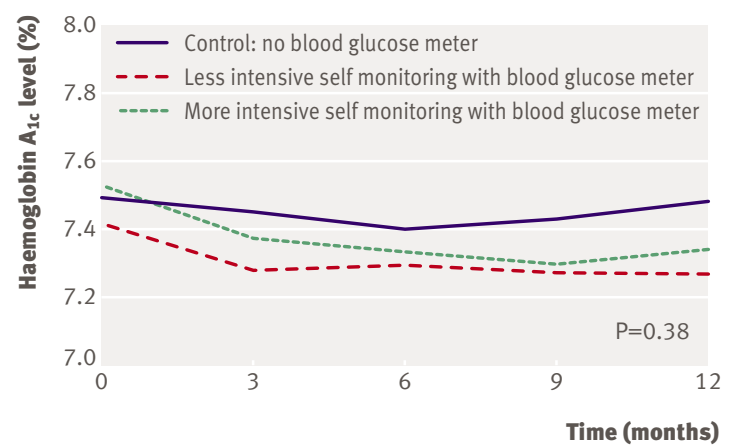

Fig 2 | Change in $\mathrm{HbA}_{1 \mathrm{c}}$ levels over 12 months' follow-up of patients with non-insulin treated type 2 diabetes according to randomisation group

Table 2 | Changes in haemoglobin $A_{1 c}$ levels, weight, and body mass index between baseline and one year in patients with non-insulin treated type 2 diabetes, by randomisation group. Values are means (standard deviations) unless stated otherwise

\begin{tabular}{|c|c|c|c|c|}
\hline Variable & $\begin{array}{l}\text { Control group* } \\
\quad(n=152)\end{array}$ & $\begin{array}{l}\text { Meter group, less } \\
\text { intensive self } \\
\text { monitoring } \\
(n=150)\end{array}$ & $\begin{array}{l}\text { Meter group, more } \\
\text { intensive self } \\
\text { monitoring } \\
(n=151)\end{array}$ & $\begin{array}{c}\text { P value for } \\
\text { difference between } \\
\text { groups } \dagger\end{array}$ \\
\hline \multicolumn{5}{|l|}{$\mathrm{HbA}_{1 \mathrm{c}}(\%):$} \\
\hline Baseline & 7.49 (1.09) & $7.41(1.02)$ & 7.53 (1.12) & 0.12 \\
\hline Follow-up & $7.49(1.20)$ & $7.28(0.88)$ & $7.36(1.05)$ & \\
\hline Change & $-0.00(1.02)$ & $-0.14(0.82)$ & $-0.17(0.73)$ & \\
\hline
\end{tabular}

\section{Systolic blood}

pressure $(\mathrm{mm} \mathrm{Hg})$ :

\begin{tabular}{|c|c|c|c|c|}
\hline Baseline & 140 (18) & 141 (17) & 137 (18) & 0.77 \\
\hline Follow-up & $136(18)$ & 137 (17) & $134(17)$ & \\
\hline Change & $-4(14)$. & $-3(16)$ & $-3(14)$ & \\
\hline
\end{tabular}

\section{Diastolic blood}

pressure $(\mathrm{mm} \mathrm{Hg})$ :

\begin{tabular}{lcccc}
\hline Baseline & $80(10)$ & $80(10)$ & $78(10)$ & 0.67 \\
\cline { 1 - 3 } Follow-up & $77(10)$ & $78(10)$ & $76(10)$ & \\
\cline { 1 - 3 } Weight $(\mathrm{kg}):$ & $-3(9)$ & $-2(9)$ & $-2(8)$ & \\
\hline Baseline & & & & \multirow{2}{*}{0.37} \\
\hline Follow-up & $86.7(18.9)$ & $90.4(18.9)$ & $86.9(16.4)$ & \\
\hline Change & $86.4(19.4)$ & $89.9(19.0)$ & $86.1(15.7)$ & $-0.8(3.3)$ \\
\hline
\end{tabular}

\section{Total cholesterol}

level $(\mathrm{mmol} / \mathrm{l})$ :

\begin{tabular}{lcccc}
\hline Baseline & $4.73(1.02)$ & $4.64(1.11)$ & $4.67(1.07)$ & 0.010 \\
\cline { 1 - 4 } Follow-up & $4.56(1.03)$ & $4.42(0.95)$ & $4.28(0.84)$ & \\
\cline { 1 - 4 } Change & $-0.16(0.84)$ & $-0.22(0.93)$ & $-0.40(0.90)$ & \\
\hline
\end{tabular}

\section{Ratio of total}

cholesterol to high

density lipoprotein

cholesterolł:

\begin{tabular}{|c|c|c|c|c|}
\hline Baseline & $4.33(1.12)$ & $4.40(1.33)$ & $4.48(1.35)$ & 0.013 \\
\hline Follow-up & $4.18(1.12)$ & $4.11(1.17)$ & $4.02(1.17)$ & \\
\hline Change & $-0.15(0.72)$ & $-0.29(0.86)$ & $-0.46(0.91)$ & \\
\hline \multicolumn{5}{|c|}{ Body mass index: } \\
\hline Baseline & $30.9(6.1)$ & $31.9(6.2)$ & $31.0(5.3)$ & 0.41 \\
\hline Follow-up & $30.8(6.3)$ & $31.8(6.3)$ & $30.7(5.0)$ & \\
\hline Change & $-0.1(1.0)$ & $-0.2(0.9)$ & $-0.3(1.2)$ & \\
\hline
\end{tabular}

Change is measured as one year follow-up minus baseline values.

*No use of blood glucose meter.

†Adjustment for baseline values.

$\ddagger$ Based on 414 participants with paired values $(137 / 152,136 / 150,141 / 151)$. checked by the research nurses using a test aliquot at baseline and at six months.

Data on adverse reactions or complications were collected at each study visit, together with information on the use of drugs.

\section{Delivery of intervention}

Training and support for the research nurses delivering the intervention was designed to ensure adherence to the study protocol..$^{15}$ The nurses were taught psychological theory and trained in behaviour change techniques and skills in delivering the intervention (six days of case based training over five weeks). Intervention protocols included scripts of the topics to be covered to guide the nurses in talking to patients. Additional measures to ensure adherence to the intervention protocols included self review of taped consultations by the research nurses and external review by a sociologist. Prompts were also built into the patient diaries to help patients adhere to their allocated intervention.

\section{Statistical analysis}

The trial was designed to have a $90 \%$ power to detect a difference of $0.5 \%$ in $\mathrm{HbA}_{1 \mathrm{c}}$ levels as the primary end point at a two sided significance level of $\mathrm{P}<0.05$. We estimated the standard deviation of $\mathrm{HbA}_{1 \mathrm{c}}$ levels to be $1.5 \%$ based on a previous trial of patients with type 2 diabetes, ${ }^{16}$ and assumed a $10 \%$ loss to follow-up. We required a total of 630 patients to achieve the specified statistical power. Subsequently we revised the estimated standard deviation for $\mathrm{HbA}_{1 \mathrm{c}}$ levels to $1.25 \%$ when it became clear that it had been overestimated. We retained a $10 \%$ dropout rate and $90 \%$ power and revised the recruitment target to 450 patients.

We carried out a single intention to treat analysis of the main trial end points at the end of the study using analysis of covariance to compare mean levels of $\mathrm{HbA}_{1 \mathrm{c}}$ at follow-up between the three allocated groups, with the baseline level of $\mathrm{HbA}_{1 \mathrm{c}}$ as a covariate. If no follow-up data were available we imputed values by carrying forward the last available measurement. We specified that in the event of a statistically significant overall result, comparisons of the two self monitoring groups independently with the control group would be carried out using $t$ tests. Levels of $\mathrm{HbA}_{1 \mathrm{c}}$ over the course of the trial were compared between groups using repeated measures analysis of variance. We also estimated the intervention effect in prespecified subgroups defined at baseline as duration of diabetes (above or below median), current management (oral hypoglycaemic drugs or dietary management only), health status (above or below the median EQ$5 \mathrm{D}$ score), and presence or absence of diabetes related complications. We tested for effect modification using analysis of covariance.

A Kaplan-Meier plot was used to explore adherence to a minimal level of self monitoring, defined as at least 26 tests over three months (equivalent to two tests each week); significance was assessed with a log rank test. The mean numbers of tests by patients carrying out at least 26 tests in each quarter are also reported, with differences between the less intensive and more 
Table 3 Changes in haemoglobin $A_{1 c}$ levels (\%) between baseline and one year by subgroup of patients with non-insulin treated type 2 diabetes. Values are means (standard deviations)

\begin{tabular}{lccc}
$\begin{array}{l}\text { Variable } \\
\text { Duration of diabetes } f\end{array}$ & $\begin{array}{c}\text { Meter group, less } \\
\text { intensive self } \\
\text { monitoring }(n=150)\end{array}$ & $\begin{array}{c}\text { Meter group, more } \\
\text { intensive self } \\
\text { monitoring }(n=151)\end{array}$ & $\begin{array}{c}\text { P value for } \\
\text { interaction† }\end{array}$ \\
\hline$\leq 36$ months: & & & $7.41(1.03)$ \\
\hline Baseline & $7.29(1.02)$ & $7.35(1.02)$ & $7.25(1.01)$ \\
\hline Follow-up & $7.30(1.24)$ & $7.23(0.93)$ & $-0.16(0.73)$ \\
\hline Change & $0.01(1.03)$ & $-0.12(0.85)$ & $7.67(1.20)$ \\
\hline s6 months: & & & $7.49(1.08)$ \\
\hline Baseline & $7.70(1.13)$ & $7.48(1.02)$ & $-0.18(0.73)$ \\
\hline Follow-up & $7.70(1.11)$ & $7.33(0.84)$ & \\
\hline Change & $-0.01(1.01)$ & $-0.15(0.80)$ & \\
\hline
\end{tabular}

\section{Baseline therapy}

\begin{tabular}{llll}
\hline Diet only: & \multicolumn{3}{l}{0.90} \\
\hline Baseline & $7.18(0.98)$ & $6.85(0.66)$ & $7.18(1.11)$ \\
\hline Follow-up & $7.21(1.05)$ & $6.90(0.70)$ & $7.09(0.94)$ \\
\hline Change & $0.03(0.80)$ & $0.04(0.64)$ & $-0.09(0.72)$ \\
\hline Oral drug therapy: & & & \\
\hline Baseline & $7.61(1.11)$ & $7.61(1.05)$ & $7.66(1.10)$ \\
\hline Follow-up & $7.61(1.24)$ & $7.41(0.91)$ & $7.46(1.07)$ \\
\hline Change & $-0.01(1.10)$ & $-0.20(0.87)$ & $-0.20(0.73)$ \\
\hline
\end{tabular}

Health status (EQ-5D)§:

Diabetes $>36$ months: 0.63

\begin{tabular}{llll}
\hline Baseline & $7.38(1.02)$ & $7.30(0.96)$ & $7.57(1.21)$ \\
\hline Follow-up & $7.46(1.16)$ & $7.22(0.76)$ & $7.43(1.16)$ \\
\hline Change & $0.07(0.99)$ & $-0.08(0.84)$ & $-0.13(0.77)$ \\
\hline Diabetes $\leq 36$ months: & & & \\
\hline Baseline & $7.54(1.16)$ & $7.50(1.09)$ & $7.34(0.80)$ \\
\hline Follow-up & $7.43(1.22)$ & $7.37(1.04)$ & $7.14(0.78)$ \\
\hline Change & $-0.11(1.14)$ & $-0.13(0.80)$ & $-0.20(0.67)$
\end{tabular}

Diabetes related complications

\begin{tabular}{llll}
\hline Absent: & \multicolumn{3}{l}{0.86} \\
\hline Baseline & $7.53(1.11)$ & $7.51(1.09)$ & $7.71(1.19)$ \\
\hline Follow-up & $7.48(1.16)$ & $7.32(0.92)$ & $-0.28(1.13)$ \\
\hline Change & $-0.05(1.02)$ & $-0.19(0.88)$ & \\
\hline Present: & & & $7.00(0.64)$ \\
\hline Baseline & $7.32(1.02)$ & $7.07(0.63)$ & $7.16(0.73)$ \\
\hline Follow-up & $7.52(1.34)$ & $7.12(0.73)$ & $0.16(0.56)$ \\
\hline Change & $0.20(1.02)$ & $0.05(0.56)$ & \\
\hline
\end{tabular}

*No use of blood glucose meter.

†After adjustment for baseline values.

$\ddagger$ Median 36 months.

§EQ-5D score 0.814. Paired data for EQ-5D score available for 384 patients.

intensive intervention groups compared with a repeated measures analysis of variance.

\section{RESULTS}

Between January 2003 and December 2005, 453 patients with non-insulin treated type 2 diabetes from 48 practices in Oxfordshire and South Yorkshire were randomised to one of three interventions (fig 1): usual care $(\mathrm{n}=152)$, less intensive self monitoring, using a blood glucose meter and advice to contact doctor for interpretation of the results $(\mathrm{n}=150)$, and more intensive self monitoring, with a blood glucose meter and training in interpreting the results $(\mathrm{n}=151)$. The median (range) number of patients per practice recruited in 24
Oxfordshire practices was 9 (2-24) and in 24 South Yorkshire practices was 8 (3-16).

Baseline personal and clinical characteristics were well balanced between the groups (table 1). The median (interquartile) duration of diabetes was 3.0 years (1.8-6.4 years), mean (SD) age was 65.7 (10.2) years, and mean (SD) level of haemoglobin $\mathrm{A}_{1 \mathrm{c}}$ was $7.5 \%$ (1.1). Only $57(12.6 \%)$ patients were lost to follow-up, which did not differ between groups (fig 1). Measurements for high density lipoprotein cholesterol levels were not obtained for 39 patients at baseline. At follow-up, $\mathrm{HbA}_{1 \mathrm{c}}$ measurements were not collected for two patients, blood pressure for five, cholesterol levels for 10 , and high density lipoprotein cholesterol levels for 15 .

\section{Primary outcome}

Table 2 shows the main results. At 12 months no difference was found in $\mathrm{HbA}_{1 \mathrm{c}}$ levels between the groups after adjustment for baseline $\mathrm{HbA}_{1 \mathrm{c}}$ levels $(\mathrm{P}=0.12)$. The mean difference in change in $\mathrm{HbA}_{1 \mathrm{c}}$ levels from baseline to 12 months between the control group and less intensive intervention group (not adjusted for baseline $)$ was $-0.14 \% \quad(95 \%$ confidence interval $-0.35 \%$ to $0.07 \%$ ) and between the control group and more intensive intervention group was $-0.17 \%$ $(-0.37 \%$ to $0.03 \%)$. Figure 2 shows the change in $\mathrm{HbA}_{1 \mathrm{c}}$ levels over the 12 months of follow-up, with no evidence of differences in levels between groups over the period of follow-up $(\mathrm{P}=0.38)$.

\section{Secondary outcomes}

A significant difference was found in the change in total cholesterol levels between the three groups $(\mathrm{P}=0.010)$. The mean difference in change in total cholesterol levels from baseline to 12 months between the control group and less intensive intervention group (not adjusted for baseline) was $-0.06 . \mathrm{mmol} / 1$ ( -0.26 to 0.14 ) and between the control group and more intensive intervention group was $-0.23(-0.43$ to -0.04$)$. No differences were found in the other secondary outcome measures (table 2). Within the prespecified subgroups no significant interactions were found with allocated group (table 3).

\section{Hypoglycaemia}

During the trial one or more grade 2 hypoglycaemic episodes were experienced by 14 patients in the control group, 33 in the less intensive intervention group, and 43 in the more intensive intervention group $\left(\chi_{2}^{2}=18.3, \mathrm{P}<0.001\right)$. Only one patient in the control group experienced a grade 3 hypoglycaemic episode.

\section{Use of meter}

Patients allocated to less intensive self monitoring were significantly more likely to persist with use of the meter than those allocated to more intensive self monitoring. Ninety nine $(67 \%)$ of those receiving the less intensive intervention and $79(52 \%)$ of those receiving the more intensive intervention continued to use the meter at least twice a week for the 12 months of the study $(\mathrm{P}=0.012$; fig 3). Among those who continued to use a 


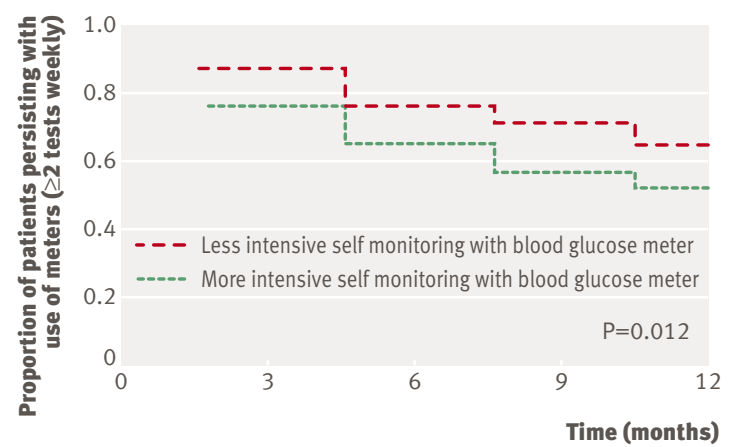

Fig $3 \mid$ Adherence to minimal level of self monitoring of blood glucose levels using a meter

meter, the mean number of readings over 12 months was significantly higher among patients receiving the more intensive intervention compared with those receiving the less intensive intervention $(\mathrm{P}=0.022$; fig 4). Eight patients in the control group started using self monitoring of blood glucose.

\section{Changes in hypoglycaemic and lipid lowering drugs} No differences were found between the groups in the proportions of patients prescribed an increase in hypoglycaemic drugs between baseline and 12 months. In the control group $45(30 \%)$ patients had increased drugs compared with $43(29 \%)$ in the less intensive intervention group and $48(32 \%)$ in the more intensive intervention group. One patient in the control group, four in the less intensive intervention group, and five in the more intensive intervention group were using insulin therapy by 12 months. No differences were found between groups in the proportions of patients where hydroxymethyl glutaryl coenzyme A reductase inhibitor (statin) treatment was increased or added to therapy. Overall, $17(11 \%)$ patients in the control group, $11(7 \%)$ in the less intensive intervention group, and $19(13 \%)$ in the more intensive intervention group who were not taking a statin at baseline were taking a statin by 12 months.

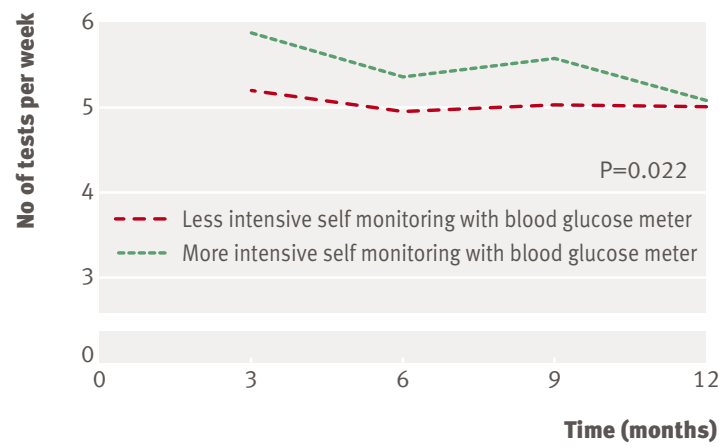

Fig 4 | Frequency of self monitoring of blood glucose levels using a meter, by randomisation group

\section{Further analyses}

Later papers will report on quality of life, cost effectiveness, and subgroup and more detailed multivariate analyses.

\section{DISCUSSION}

No significant improvement in glycaemic control was found after 12 months in patients with non-insulin treated type 2 diabetes using self monitoring of blood glucose levels when compared to those not self monitoring. No evidence was found of a significantly different impact of self monitoring on glycaemic control when comparing subgroups of patients defined by duration of diabetes, therapy, diabetes related complications, and EQ-5D score. Also no evidence was found that more intensive compared with less intensive monitoring led to differences in glycaemic control.

\section{Strengths and weaknesses of the study}

In this study patients were independently randomised, with concealed allocation of measurement of the main outcome and a low loss to follow-up. Participants were drawn from a well defined sampling frame and the reasons for exclusion were fully recorded. Recruitment targets were revised after baseline data on haemoglobin $\mathrm{A}_{1 \mathrm{c}}$ levels in the first 245 randomised patients indicated that the standard deviation had been overestimated in the original power calculations. ${ }^{9} \mathrm{We}$ did not, however, change the proposed power or significance levels. Participants' diabetes was reasonably well controlled and although most were not using a meter a minority had had experience of their use. Both these factors may have limited scope for further improvement in glycaemic control. However, the participants were representative of well controlled noninsulin treated patients with type 2 diabetes in the community who are the target group for current recommendations of up to twice daily self monitoring and testing after meals. ${ }^{17} 18$

Designing a trial to evaluate self monitoring of blood glucose levels is complex because it must include an educational component on the use and interpretation of testing for the intervention group,${ }^{19}$ whereas advice on improving self care must be offered to the comparison group. ${ }^{3}$ We tackled these issues by providing a common structure for interventions, incorporating standardised good care in all three arms of the trial within which nurses discussed issues of glycaemic control, assessed either by $\mathrm{HbA}_{1 \mathrm{c}}$ levels or self monitoring of blood glucose, and its role in setting and monitoring self care goals. ${ }^{9}$ The stepwise approach to the interventions across the three arms of the trial allowed examination of what aspects of the intervention, if any, were responsible for improved outcomes. Recent consensus guidelines have based recommendations for self monitoring of blood glucose levels on a theoretical potential to better self manage glycaemic control. ${ }^{202}$ We incorporated self monitoring of blood glucose into a framework that, based on psychological theory, should have optimised its effect. Careful specification, training, and monitoring of consultations ensured that the allocated interventions were delivered as 
planned, ${ }^{22}$ although some patients in the less intensive intervention group may themselves have adopted a more intensive monitoring approach. Despite an intervention based on standards of best clinical practice and underpinned by appropriate psychological theory, we found no convincing evidence of an effect on glycaemic control.

\section{Strengths and weaknesses in relation to other studies}

Comparisons with early trials of blood glucose monitoring are of limited relevance because of their small size, the large quantity of blood required to be read by older meters, and the skill required for their use. However, more recent trials have been carried out with meters utilising technologies that require smaller amounts of blood and simplified procedures for testing. Our findings support those of a recent small trial using standardised counselling for both intervention and control groups. The trial reported a non-significant reduction in $\mathrm{HbA}_{1 \mathrm{c}}$ levels of $0.2 \%$ in the intervention group compared with the control group. ${ }^{23}$ Our findings, however, conflict with the findings of two of the largest trials of self monitoring of blood glucose to date. One of these trials reported a significant decrease in $\mathrm{HbA}_{1 \mathrm{c}}$ level of $0.3 \%$ in the intervention group compared with the control group. ${ }^{24}$ However, over 30\% of those randomised were lost to follow-up and missing values were not imputed, which might lead to bias. In addition, initial specific training in use of a blood glucose meter was not matched by additional training for the control group, although all patients received dietary advice regardless of randomisation. A second trial reported a reduction in $\mathrm{HbA}_{1 \mathrm{c}}$ level of $0.46 \%$ in the intervention group compared with the control group. Standardised counselling supporting lifestyle modification was, however, only provided to the self monitoring group. ${ }^{25}$ This type of educational support for self management in itself has been estimated as improving $\mathrm{HbA}_{1 \mathrm{c}}$ levels by $0.26 \%{ }^{26}$

\section{Meaning of the study}

Fewer people in our trial allocated to more intensive self monitoring compared with less intensive self monitoring continued testing; previous studies have found that trying to understand blood glucose measurements may lead to frustration when results do not fall into a pattern, or cease to be of interest when they are entirely predictable. ${ }^{27}$ Patients with reasonably well controlled diabetes do not need active encouragement to use a meter. The increased recording of hypoglycaemia in the self monitoring arms may be a result of an increased awareness of low blood glucose levels from using the meter rather than a true biochemical difference between groups. Although no improvement in glycaemic control was observed, a small but significant improvement was found in total cholesterol levels with the self monitoring intervention. This finding is consistent with an increased intensity of self management in these groups, possibly mediated through increased dietary adherence or through taking lipid lowering drugs more regularly.
Unanswered questions and future research

Recent systematic reviews have estimated a benefit of $0.4 \%$ from self monitoring, ${ }^{2}$ and on this basis a previous study has estimated an incremental cost of $£ 4500$ ( $€ 6650 ; \$ 8880)$ to $£ 15515$ per quality of life year gained. Our estimates of the size of effect on $\mathrm{HbA}_{1 \mathrm{c}}$ levels suggest that it is probable that the previous study underestimated the cost per quality of life year gained. A comprehensive economic evaluation with cost effectiveness estimates will be detailed in a future report.

Evidence of benefit from self monitoring of blood glucose for other patient groups is stronger. Large trials of management of patients with type 1 diabetes have incorporated self monitoring of blood glucose as an essential part of self management. ${ }^{2829}$ Self monitoring for insulin treated patients with type 2 diabetes is accepted practice, although the evidence base requires further work and optimisation of its use may be possible. However, routine self monitoring of blood glucose for patients with reasonably well controlled non-insulin treated type 2 diabetes seems to offer, at best, small advantages; is not well accepted; and the cost, effort, and time involved in the procedures may be better directed to supporting other health related behaviours. Current guidelines for the use of self monitoring of blood glucose among patients with reasonably well controlled non-insulin treated type 2 diabetes should be reviewed.

We thank the patients who took part in this study and their doctors for support and help. W Hardeman and I Hobbis contributed to the development of the intervention protocols and prepared and led some of the training sessions for the nurses. M McKinnon and J Donnelly helped train the nurses and L Rosmovitz carried out external review of interventions.

Contributors: $A F, A-L K$, and $A N$ had the original idea for the study and wrote the trial protocol with PY, DF, and RH. AF, AW, DF, and A-LK developed the trial measures and intervention. PY was trial statistician and analysed the data. AW, $A F, A C$, and $E G$ managed the trial. AF wrote the first draft of the manuscript with $A N$ and $A-L K$ and all members of the writing group reviewed and commented on the final manuscript. AF is guarantor of this paper. The DiGEM Trial Group. Writing committee: AF, AW, EG, PY, DF, AC, RH, A-LK, and AN. Investigators: AF, AN, A-LK, D Mant, S Ziebland, DF, A Gray, PY, and RH. Steering committee: N Stott (chair), AF, AN (to 2005), S Sutton, H Tewson, D Chapman, H Hearnshaw, E Goyder (from 2005), P Glasziou (from 2005), M Jiwa (2004 to 2005), and M Gordon (from 2005). Intervention development: AW, AF, DF, A-LK, and MP Selwood. Coordinating Centres: (Oxford) AW (to 2005, trial coordinator), AC (trial manager), PY (trial statistician), J Simon (health economist), and A Fuller (data manager); (Sheffield) Vivienne Walker (local trial administrator). Data monitoring committee: C Baigent (chair), J Levy, and K Wheatley. Research nurses (Oxford) MP Selwood, H Kirlow, M Chapman, and S Turner; (Sheffield) A Casbolt, K Dobson, A Willert, A Roberts, and H Wood. Central laboratory: K Islam.

Funding: The trial was funded by the NHS and the National Insitute for Health Research health technology assessment programme. The opinions expressed in this report are not necessarily those of the Department of Health. Abbott Diabetes Care provided blood glucose meters (Optium). AF was supported by an NHS research and development career development award from 2001-5. AW was supported by a Rhodes scholarship.

Competing interests: None declared.

Ethical approval: This study was approved by the Oxfordshire Research Ethics Committee B (002.059).

1 Wild S, Roglic G, Green A, Sicree R, King H. Global prevalence of diabetes: estimates for the year 2000 and projections for 2030 . Diabetes Care 2004;27:1047-53.

2 Coster S, Gulliford MC, Seed PT, Powrie JK, Swaminatham R. Selfmonitoring in type 2 diabetes mellitus: a meta-analysis. Diabet Med 2000;17:755-61. 


\section{WHAT IS ALREADY KNOWN ON THIS TOPIC}

Self monitoring of glucose for non-insulin treated patients with diabetes is costly but may improve glycaemic control

Although some observational studies have suggested benefits, the results of randomised trials have been inconclusive

\section{WHAT THIS STUDY ADDS}

It is not necessary to routinely recommend self monitoring of blood glucose in reasonably well controlled patients with non-insulin treated type 2 diabetes

3 Welschen LMC, Bloemendal E, Nijpels G, Dekker IM, Heine RI, Stalman WAB, et al. Self-monitoring of blood glucose in patients with type 2 diabetes who are not using insulin: a systematic review. Diabetes Care 2005:28:1510-7.

4 Sarol JN Ir, Nicodemus NA Ir, Tan KM, Grava MB. Self-monitoring of blood glucose as part of a multi-component therapy among noninsulin requiring type 2 diabetes patients: a meta-analysis (19662004). Curr Med Res Opin 2005:21:173-84

5 Karter Al, Parker MM, Moffet HH, Spence MM, Chan J, Ettner SL, et al. Longitudinal study of new and prevalent use of self-monitoring of blood glucose. Diabetes Care 2006;29:1757-63.

6 Martin S, Schneider B, Heinemann L, Lodwig V, Kurth J, Kolb H, et al. Self-monitoring of blood glucose in type 2 diabetes and long-term outcome: an epidemiological cohort study. Diabetologia 2006;49:271-8.

7 Farmer Al, Neil A. Variations in glucose self-monitoring during oral hypoglycaemic therapy in primary care [letter]. Diabet Med 2004:22:511.

8 Davidson MB. Counterpoint: self-monitoring of blood glucose in type 2 diabetic patients not receiving insulin: a waste of money. Diabetes Care 2005:28:1531-3.

9 Farmer A, Wade A, French DP, Goyder E, Kinmonth AL, Neil A. The DiGEM trial protocol—a randomised controlled trial to determine the effect on glycaemic control of different strategies of blood glucose self-monitoring in people with type 2 diabetes [ISRCTN47464659]. BMC Fam Pract 2005;6:25.

10 EuroQol Group. EuroQol: a new facility for the measurement of health related quality of life. Health Pol 2001;16:199-208.

11 Leventhal H, Nerenz DR, Steele DJ. Illness representations and coping with health threats. In: Baum A, Taylor SE, Singer JE, eds. Handbook of psychology and health. Hillsdale, NJ: Erlbaum, 1984:219-52.

12 Hardeman W, Griffin S, Johnston M, Kinmonth AL, Wareham NJ. Interventions to prevent weight gain: a systematic review of psychological models and behaviour change methods. Int J Obes Relat Metab Disord 2000;24:131-43.

13 Strecher VI, Seijts GH, Kok GJ, Latham GP, Glasgow R, DeVellis B, et al. Goal setting as a strategy for health behavior change. Health Educ Q 1995;22:190-200.

14 National Institute for Clinical Excellence. Management of type 2 diabetes: management of blood glucose. London: NICE, 2002.

15 Hardeman W, Sutton S, Griffin S, Johnston M, White AJ, Wareham NJ, et al. A causal modelling approach to the development of theory- based behaviour change programmes for trial evaluation. Health Educ Res 2005;20:676-87.

16 Turner RC, Cull CA, Frighi V, Holman RR. Glycemic control with diet, sulfonylurea, metformin, or insulin in patients with type 2 diabetes mellitus: progressive requirement for multiple therapies (UKPDS 49). UK Prospective Diabetes Study (UKPDS) Group. JAMA 1999;281:2005-12.

17 Bergenstal RM, Gavin III JR. The role of self-monitoring of blood glucose in the care of people with diabetes: report of a global consensus conference. Am / Med 2005;118(9, suppl 1):1-6.

18 Gerich JE. Clinicians can help their patients control postprandial hyperglycemia as a means of reducing cardiovascular risk. Diabetes Educator 2006;32:513-22.

19 Franciosi M, Pellegrini F, De Berardis G, Belfiglio M, Di Nardo B, Greenfield S, et al. Self-monitoring of blood glucose in non-insulintreated diabetic patients: a longitudinal evaluation of its impact on metabolic control. Diabet Med 2005;22:900-6.

20 Owens DR, Barnett AH, Pickup J, Kerr D, Bushby P, Hicks D, et al. Blood glucose self-monitoring in type 1 and type 2 diabetes: reaching a multi-disciplinary consensus. Diabetes Primary Care 2004;6:398-402.

21 American Diabetes Association. Standards of medical care in diabetes-2006. Diabetes Care 2006;29(suppl 1):S4-42.

22 Bellg AJ, Borrelli B, Resnick B, Hecht J, Minicucci DS, Ory M, et al. Enhancing treatment fidelity in health behavior change studies: best practices and recommendations from the $\mathrm{NIH}$ Behavior Change Consortium. Health Psychol 2004:23:443-51.

23 Davidson MB, Castellanos M, Kain D, Duran P. The effect of self monitoring of blood glucose concentrations on glycated hemoglobin levels in diabetic patients not taking insulin: a blinded, randomized trial. Am / Med 2005;118:422-5.

24 Guerci B, Drouin P, Grange V, Bougneres P, Fontaine P, Kerlan V, et al. Self-monitoring of blood glucose significantly improves metabolic control in patients with type 2 diabetes mellitus: the autosurveillance intervention active (ASIA) study. Diabetes Metab 2003;29:587-94.

25 Schwedes U, Siebolds M, Mertes G. Meal-related structured selfmonitoring of blood glucose: effect on diabetes control in noninsulin-treated type 2 diabetic patients. Diabetes Care 2002;25:1928-32.

26 Norris SL, Lau J, Smith SI, Schmid CH, Engelgau MM. Selfmanagement education for adults with type 2 diabetes: a metaanalysis of the effect on glycemic control. Diabetes Care 2002;25:1159-71.

27 Peel E, Parry O, Douglas M, Lawton J. Blood glucose self-monitoring in non-insulin-treated type 2 diabetes: a qualitative study of patients' perspectives. Br J Gen Pract 2004;54:183-8.

28 Diabetes Control and Complications Trial/Epidemiology of Diabetes Interventions and Complications (DCCT/EDIC) Study Research Group. Intensive diabetes treatment and cardiovascular disease in patients with type 1 diabetes. N Engl J Med 2005;353:2643-53.

29 DAFNE Study Group. Training in flexible, intensive insulin management to enable dietary freedom in people with type 1 diabetes: dose adjustment for normal eating (DAFNE) randomised controlled trial. BMJ 2002;325:746.

Accepted: 13 June 2007 